\title{
Proprioception and Balance as Predictors of Ankle Injuries Among NCAA Student Athletes
}

\author{
Ashley B. Stiltner ${ }^{1}$, Haley Felts ${ }^{1, ~}$, Kenneth D. Royal ${ }^{2}$ \\ ${ }^{1}$ College of Arts and Sciences, King University, Bristol, TN, USA \\ ${ }^{2}$ Department of Clinical Sciences, North Carolina State University, Raleigh, NC, USA
}

Email address:

kdroyal2@ncsu.edu (H. Felts)

${ }^{*}$ Corresponding author

\section{To cite this article:}

Ashley B. Stiltner, Haley Felts, Kenneth D. Royal. Proprioception and Balance as Predictors of Ankle Injuries Among NCAA Student Athletes. International Journal of Sports Science and Physical Education. Vol. 2, No. 4, 2017, pp. 53-56. doi: 10.11648/j.ijsspe.20170204.11

Received: July 7, 2017; Accepted: July 17, 2017; Published: August 11, 2017

\begin{abstract}
Ankle sprains are common in sports and account for nearly $15 \%$ of all sports-related injuries. Ankle injuries often result in functional instability of the ankle that makes these injuries susceptible to recurring. Extant research has noted that functional instability of the ankle does not result from mechanical hypermobility, but instead from a loss of neuromuscular control. Thus, it seems proprioception and balance could be a predictor of ankle sprain. Given the costs of ankle injuries for collegiate athletes (e.g., painful, potentially recurring, expensive to treat, result in loss of playing time, and may potentially impact a student athlete's potential for a career as a professional athlete), this study sought to determine if various factors such as gender, sport played, history of balance problems, self-perception of ankle stability, and the result of a modified Romberg Test could predict ankle sprain among 128 NCAA Division II student athletes.
\end{abstract}

Keywords: Balance, Student Athletes, NCAA, Ankle Injury, Athletic Training, Sports Medicine, Athletic Injuries

\section{Introduction}

Ankle sprains are among the most common injuries that plague athletes, [1] and estimates suggests the injuries account for nearly $15 \%$ of all sports related injuries. [2] Ankle injuries often result in recurrent injury and leads to functional instability of the ankle. [3,4] According to extant research, functional instability of the ankle does not result from mechanical hypermobility, but instead from a loss of neuromuscular control. [5] It has been determined that patients with a history of multiple ankle sprains have an increase of postural sway and ankle repositioning errors leading to an increased risk of recurrent injury. [6, 7] Further, injury to the ligaments of the ankle is one of the most common injuries sustained during athletic activities. [8] These injuries can be expensive to treat, lead to an early on-set of osteoarthritis, [9], result in decreased physical activity, [10-12] and affect one's physical quality of life. [13-16]. For an athlete, however, the cost the injury can be even greater. There typically is much focus on treating the immediate symptoms, but little thought is given to the long-term effects this injury will have on the athlete's neuromuscular control and function. As a result, neuromuscular control is never fully regained and leaves the athlete much more susceptible to recurrent injury. Given this backdrop, the purpose of this study was to determine if proprioception and balance could be a predictor of ankle sprain in collegiate level athletes.

\section{Methods}

\subsection{Participants}

A total of 128 NCAA Division II student athletes at a small university in the southeastern United States comprised the sample frame for the study. Of the 128 athletes, $72(56.3 \%)$ were male and $56(43.85 \%)$ were female. Student athletes participated in the following sports: Baseball, Basketball, Cycling, Cheerleading, Golf, Soccer, Softball, Swimming, Track \& Field, Volleyball and Wrestling.

\subsection{Measures}

A Romberg's test is a well-evidenced instrument commonly used to identify deficits in standing balance in both 
clinical and research settings. [17-20] In these screening tests, subjects are observed for any signs of imbalance including swaying, inability to maintain proper position, or balance checks. If subjects fail at any point they are not permitted to continue with testing as this could pose a danger to them. For the present study, a modified Romberg Test of Standing Balance on Firm and Compliant Support Surfaces was used to examine subjects' ability to stand unassisted under four test conditions, ordered in increasing level of difficulty.

Two initial Romberg's test were used as screening instruments to identify the possibility of any underlying cerebellar issues that may render the subjects unable to undergo further, more difficult stages of testing. In test \# 1, subjects stood fully upright making use of all the sensory inputs that contribute to balance (e.g, the central vestibular system, vision, and proprioception). In test \# 2, subjects closed their eyes to eliminate any visual input so that balance was tested when only vestibular and proprioceptive information was available. In test \# 3, subjects must maintain balance on a BOSU ball, which reduces the proprioceptive input, leaving only visual and vestibular cues. In test \# 4, also performed while standing on a BOSU ball, subjects' eyes were closed to remove visual input, and subjects' ability to maintain balance was tested using only the vestibular system.

Prior to testing, a survey also was administered to each student athlete. The purpose of the survey was to inform the principle investigator of any contraindications to testing, as well as solicit additional insights about the injury history of each student athlete. The survey included a number of items inquiring about student athletes' current health, sport played, previous ankle injuries, methods used to treat previous injuries, self-reported lost playing time due to previous ankle injury, history of balance problems, and a self-reported measure of perceived ankle stability.

\subsection{Setting}

In the Fall of 2014, the university's Institutional Review Board (IRB) approved the present study. In the Spring and Fall of 2015, the modified Romberg tests were administered at the NCAA mandated athletic physicals. This timing permitted access to as many athletes as possible in an environment conducive to this type of testing.

\subsection{Procedures}

During the physical examinations, a station was set up at the end of the exam. First, students were provided informed consent for their information to be used as part of this research Students electing to take part in the study signed the consent form and then completed the aforementioned informational survey. Upon completion of the survey, the modified Romberg testing began. Specifically, athletes were tested with their eyes open and then closed. Balance was tested using the Dyna Disc Balance Disc, which provides an unstable standing surface requiring the subject to engage active proprioceptors to maintain their balance. Subjects' test results were recorded, and athletes were monitored as they normally are by the athletic training staff at the university. At the end of the Fall 2015 and Spring 2016 semesters, the athletic training staff were contacted and asked to provide information about any athlete who either reported an ankle injury or was treated for an ankle injury during their respective sport season.

\subsection{Data Analysis}

Data analyses involved calculating a variety of descriptive statistics and chi-squared tests. SPSS statistical software (version 24) was used to perform the analyses. All significance testing was performed using $95 \%$ confidence levels.

\section{Results}

Student athletes' responses to the survey items are presented in Table 1. Results indicate most student athletes had previously experienced an ankle sprain (57.5\%), but only a relative few had experienced multiple sprains. Only 9 (7.1\%) athletes had previous balance training, and only 2 $(1.7 \%)$ individuals reported having a history of balance problems. With respect to ankle stability, most athletes $(\mathrm{n}=$ $98,83.1 \%$ ) reported to have no problems with ankle stability.

Table 1. Student athletes' responses to survey items.

\begin{tabular}{ll}
\hline Variable & N (\%) \\
\hline History of sprained ankle & $73(57.5 \%)$ \\
Yes & $54(42.2 \%)$ \\
No & \\
Number of previous sprained ankles* & $2(4.2 \%)$ \\
0 & $24(50.0 \%)$ \\
1 & $8(16.7 \%$ \\
2 & $5(10.4 \%)$ \\
3 & $2(4.2 \%)$ \\
4 & $4(8.3 \%)$ \\
5 & $3(6.3)$ \\
6 or more & \\
Previous balance training & $9(7.1 \%)$ \\
Yes & $118(92.9 \%)$ \\
No & \\
History of balance problems & $2(1.7 \%)$ \\
Yes & $115(98.3 \%)$ \\
No & \\
Unstable ankle & $6(4.7 \%)$ \\
Yes & $98(83.1 \%)$ \\
No & $14(11.9 \%)$ \\
Sometimes &
\end{tabular}

With respect to the modified Romberg tests, all 128 student athletes passed Test \# 1 and Test \# 2, 3 (2.3\%) students failed Test \# 3, and 66 (51.6\%) failed Test \#4. Overall, results of the modified Romberg tests suggest student athletes generally possessed acceptable proprioception and balance.

Because Test \# 4 was the most discerning of balance and proprioception, pass/fail results were investigated by gender, sport, number of previous ankle injuries, history of balance problems, and a self-report of ankle stability. Chi-squared tests indicated no statistically significant differences were discernible by gender, $\chi^{2}(1)=574, p=449$; sport played, $\chi^{2}$ 
$(21)=24.580, p=226$; number of previous ankle injuries, $\chi^{2}$ (6) $=7.351, p=290$; history of balance problems, $\chi^{2}(1)=$ $2.216, p=137$; or self-reported ankle stability, $\chi^{2}(2)=1.286$, $p=526$;

A total of $20(18.5 \%)$ student athletes later acquired an ankle injury during their respective sporting season. A series of chi-squared analyses were performed on students who acquired an ankle injury versus those that did not in an effort to identify any factors associated with injury presence or absence. Specifically, the following factors were tested: gender, sport, history of balance problems, self-reported ankle stability measure, and pass/fail result of Romberg Test \# 4. Chi-squared tests indicated no statistically significant differences were discernible by gender, $\chi^{2}(1)=737, p=390$; sport, $\chi^{2}(21)=25.472, p=227$; history of balance problems, $\chi^{2}(1)=370, p=543$; ankle stability, $\chi^{2}(2)=2.809, p=246$; or Romberg Test \# 4, $\chi^{2}(1)=2.603, p=107$.

\section{Discussion}

This study sought to determine if balance and proprioception could be used as a predictor of ankle sprains in collegiate level athletes. In general, results indicate no demographic variable or health-related factor predicted performance on the modified Romberg tests. Further, the student athletes that did acquire an ankle injury during their sporting season did not differ in any systematic way from students that did not acquire an ankle injury with respect to gender, sport, history of balance problems, ankle stability, or performance on the Romberg tests. Collectively, these findings indicate that balance and proprioception are not adequate predictors of ankle injury in collegiate level athletes.

A number of previous studies, however, have found contrasting results. These include studies performed on dancers and athletes, which indicated that proprioceptive deficits of the ankles are predictors of ankle injury. [7] Given the mixed results on similar samples, additional studies are necessary. One element that may improve future studies is to also investigate subjects' medical records and previous injury reports. At present, most studies rely on self-reported data which may or may not be a contributing source of error. The inclusion of previously documented records could add a layer of objectivity, thus helping increase the validity of a study's findings. Additionally, future studies may also take into account possible variations such as footwear and previous knee, hip, or spinal injuries.

As with any study, the present study is not without some limitations. First, students were presented a survey and asked a number of questions that may be somewhat difficult for students to truly know. For example, without a diagnosis from a medical professional student athletes' may or may not accurately know their true medical history (e.g., a previous ankle sprain may have gone undiagnosed, or the suspicion of a previous ankle sprain may be considered a 'false positive'). Second, there is the possibility that students could have sustained ankle injuries and not reported them to the athletic training staff for fear of lost playing time. For many student athletes, competitive performance of sport may dictate both one's future career as a professional athlete and potential financial earnings, thus many students may be reluctant to admit injury given the stakes may be so costly.

\section{Conclusion}

During the Spring 2015 and Fall 2015 NCAA athletic screening physicals, 128 NCAA Division II collegiate level athletes were tested using a modified Romberg's Test. The first two tests were performed with athletes standing on the floor to provide a screening tool that illustrated their ability to move further with the testing and eliminated the possibility of vestibular disorders. The last two tests were performed on a Dyna Disc Balance Disc, which provides an unstable standing surface requiring the subject to engage active proprioceptors to maintain balance. Results found no significant differences among those who did sustain ankle injuries during their respective seasons and those who did not based on gender, sport, history of balance problems, self-reported ankle stability measure, and the pass/fail result of Romberg Test \# 4. These findings contrast with previous research, thus additional research is needed in this area.

\section{References}

[1] Wilkerson, G. B. (1992). Ankle injuries in athletes. Primary Care, 19, 377-392.

[2] Holme, E., Magnusson, S. P., Becher, K., et al. (1999). The effect of supervised rehabilitation on strength, postural sway, position sense and re-injury after acute ankle ligament sprain. Scandinavian Journal of Medicine \& Science in Sports, 9 (2), 104-109.

[3] Ananda coomarasamy, A., \& Barnsley, L. (2005). Long term outcomes of inversion ankle injuries. British Journal of Sports Medicine, 39: e14; discussion e14.

[4] Konradsen, L., Bech, L., Ehrenbjerg, M., et al. (2002). Seven years follow-up after ankle inversion trauma. Scandinavian Journal of Medicine \& Sciences in Sports, 12, 129-35.

[5] Richie, D. H. Jr. (2001). Functional instability of the ankle and the role of neuromuscular control: A comprehensive review. Journal of Foot Ankle Surgery, 40 (4), 240-251.

[6] De Noronha, M., Refshauge, K. M., Herbert, R. D., Killbreath, S. L., \& Hertel, J. (2006). Do voluntary strength, proprioception, range of motion, or postural sway predict occurrence of lateral ankle sprain? British Journal of Sports Medicine, 40 (10), 824-828.

[7] Leanderson, J., Eriksson, E., Nilsson, C., \& Wykman, A. (1996). Proprioception in classical ballet dancers. A prospective study of the influence of an ankle sprain on proprioception in the ankle joint. American Journal of Sports Medicine. 24 (3), 370-374.

[8] Mohammad, A., Hossein, K., Hossein, F., \& Soghrat, F. (2006) Balance problems after unilateral ankle sprains. Journal of Rehabilitation Research and Development, 43, 819-824. 
[9] Golditz T, Steib S, Pfeifer K, et al. (2014). Functional ankle instability as a risk factor for osteoarthritis: Using T2-mapping to analyze early cartilage degeneration in the ankle joint of young athletes. Osteoarthritis and Cartilage, 22, 1377-85.

[10] Hubbard-Turner, T, \& Turner, M. J. (2015). Physical activity levels in college students with chronic ankle instability. Journal of Athletic Training, 50, 742-7.

[11] Hubbard-Turner, T., Wikstrom, E. A., Guderian, S., et al. (2015). An acute lateral ankle sprain significantly decreases physical activity across the lifespan. Journal of Sports Science Medicine, 14, 556-61.

[12] Simon J. E., \& Docherty, C. L. (2014). Current health-related quality of life is lower in former Division I collegiate athletes than in non-collegiate athletes. American Journal of Sports Medicine, 42, 423-9.

[13] Arnold, B. L., Wright, C. J., \& Ross, S. E. (2011). Functional ankle instability and health-related quality of life. Journal of Athletic Training, 46, 634-41.

[14] Houston, M. N., Hoch, J. M., Gabriner, M. L., et al. (2015). Clinical and laboratory measures associated with health-related quality of life in individuals with chronic ankle instability. Physical Therapy in Sport, 16, 169-75.

[15] Houston, M. N., Hoch, J. M., \& Hoch, M. C. (2015).
Patient-reported outcome measures in individuals with chronic ankle instability: A systematic review. Journal of Athletic Training, 50, 1019-33.

[16] Houston, M. N., Van Lunen, B. L., \& Hoch, M. C. (2014). Health-related quality of life in individuals with chronic ankle instability. Journal of Athletic Training, 49, 758-63.

[17] National Institute of Health and Nutrition Examination Survey. (2001). Balance and Procedures Manual. Available at: https://www.cdc.gov/nchs/data/nhanes/ba.pdf. Accessed on July 7, 2017.

[18] Kim, S., Kim, M., Kim, N., Kim, S., \& Han, G. (2012). Quantification and validity of modified Romberg tests using three-axis accelerometers. Green and Smart Technology With Sensor Applications. Edited by: Cho H, Kim T, Mohammed S, Adeli H, Oh M, Lee K-W. Heidelberg: Springer Berlin, 254-261. Communications in Computer and Information Science, vol. 338 .

[19] Lanska, D. J., \& Goetz, C. G. (2000). Romberg's sign: Development, adoption, and adaptation in the 19th century. Neurology, 55, 1201-1206.

[20] Findlay, G. F. G., Balain, B., Trivedi, J. M., \& Jaffray, D. C. (2009). Does walking change the Romberg sign? European Spine Journal, 18, 1528-1531. 